\title{
Propellant-Free Control of Tethered Formation Flight, Part 2: Nonlinear Underactuated Control
}

\author{
Soon-Jo Chung* \\ Iowa State University, Ames, Iowa 50011 \\ and \\ Jean-Jacques E. Slotine $\$$ and David W. Miller \\ Massachusetts Institute of Technology, \\ Cambridge, Massachusetts 02139
}

DOI: $10.2514 / 1.32189$

\begin{abstract}
This is the second in a series of papers that exploit the physical coupling of tethered spacecraft to derive a propellant-free spin-up and attitude control strategy. We take a nonlinear control approach to underactuated tethered formation flying spacecraft, whose lack of full state feedback linearizability, along with their complex nonholonomic behavior, characterizes the difficult nonlinear control problem. We introduce several nonlinear control laws that are more efficient in tracking time-varying trajectories than linear control. We also extend our decentralized control approach to underactuated tethered systems, thereby eliminating the need for any intersatellite communication. To our knowledge, this work reports the first nonlinear control results for underactuated tethered formation flying spacecraft. This article further illustrates the potential of the proposed strategy by providing a new momentum dumping method that does not use torque-generating thrusters.
\end{abstract}

\section{Introduction}

A S DISCUSSED in the first paper of this series [1], most of the previous work on tethered satellite formation flight is based upon the assumption that the tethered system is fully actuated (both thruster force $F$ and torque $u$ are available). Motivated by the controllability analysis illustrated in the first paper [1], indicating that both array resizing and spin-up are fully controllable by the reaction wheels $u$ and the tether motor, the aim of this paper is to introduce several new nonlinear control techniques for spinning tethered arrays without thrusters $(F=0)$. We exploit partial feedback linearization, feedback linearization via momentum decoupling, and backstepping, and compare the performance of nonlinear control laws with that of gain-scheduling linear control [1]. We shall consider only the case of the fixed tether length, focusing on the spin-up attitude control problem on the assumption that the tether length is controllable separately.

This paper investigates the feasibility of controlling the array spin rate and relative attitude without the use of thrusters. As stated in the first paper of this series [1] , we can dramatically increase the life span of the mission by using reaction wheels instead of thrusters for controlling the array spin rate. Also, the optics will not risk contamination by exhaust from the thrusters. The proposed underactuated method is most effective for a compact configuration with short baselines [1]. This article also fulfills the potential of the proposed strategy by providing a new momentum dumping method without the need for torque-generating thrusters; the compound pendulum mode and array spin rate are stabilized using only the

Presented as Paper 6858 at the AIAA Guidance, Navigation, and Control Conference and Exhibit, at the Marriott Hilton Head Beach and Golf Resort in Hilton Head, SC, 20-23 August 2007; received 16 May 2007; revision received 10 May 2008; accepted for publication 10 May 2008. Copyright $\odot$ 2008 by the American Institute of Aeronautics and Astronautics, Inc. All rights reserved. Copies of this paper may be made for personal or internal use, on condition that the copier pay the $\$ 10.00$ per-copy fee to the Copyright Clearance Center, Inc., 222 Rosewood Drive, Danvers, MA 01923; include the code 0731-5090/08 \$10.00 in correspondence with the CCC.

*Assistant Professor of Aerospace Engineering; sjchung@alum.mit.edu. AIAA Member.

'Professor of Mechanical Engineering and Information Sciences, Professor of Brain and Cognitive Sciences; jjs@ mit.edu.

Frofessor, Department of Aeronautics and Astronautics; millerd@ mit.edu. AIAA Senior Member. linear thruster and translational actuator on the tether during the operation of momentum dumping.

Control of underactuated mechanical systems is an active area of research [2-5]. In particular, Spong [6] developed the partial feedback linearization technique for the swing-up maneuver of the acrobot. One drawback of the partial feedback linearization method is that it does not automatically guarantee stable zero dynamics after applying the change of control. Backstepping [7] is another alternative methodology to come up with an underactuated nonlinear controller. However, backstepping is applicable only to strictfeedback systems. A model reduction technique, transforming a class of underactuated systems to cascade normal forms, is presented in [4,8]. In addition, recent work examines the sliding-mode control [9], intelligent control [10], and hybrid switching control [11] for underactuated nonlinear systems. In the context of geometric control theory, two energy-based methods can be considered for underactuated nonlinear systems. First, an oscillatory control based on averaging $[2,3]$ can be developed, which requires a highfrequency control input. The second interesting geometric control approach is the method of controlled Lagrangians via the so-called matching process [2]. In essence, the control design involves shaping the system's total or kinetic energy with the additional parameters and the matching process. One limitation is that generic physical damping makes the control-modified energy rate indefinite, thus invalidating the nonlinear stability argument of the controlled Lagrangian method [12]. Because the synchronized position hold, engage, and reorient experimental satellites (SPHERES) tethered formation flying experimental setup involves various forms of friction (see [13]), the method of controlled Lagrangians is not pursued in this paper.

Control of underactuated spacecraft has also been a popular subject. Of particular interest is work by Tsiotras et al. [14-16] showing that a nonsmooth time-invariant feedback control law can be used to rotate an axis-symmetric rigid spacecraft to the equilibrium using only two control torques. In $[17,18]$, underactuated control of a dumbbell spacecraft is studied.

Most of the aforementioned work is restricted to a single-body dynamics problem. In this paper, the decentralized control strategy from our prior work [19] is extended to the underactuated control of multivehicle tethered formation flying. To our knowledge, this work presents the first linear and nonlinear control results for underactuated tethered formation flight systems. 
The remainder of the paper is organized as follows. After reviewing some fundamental aspects of underactuated tethered systems in Sec. II, we present nonlinear control laws based on partial feedback linearization (Sec. III), feedback linearization via momentum decoupling (Sec. IV), and backstepping (Sec. V). We show in Sec. VI that a fully decentralized control law designed from the underactuated single-tethered system can stabilize a multivehicle tethered array. Section VII discusses simulation results, where the nonlinear tracking control laws are compared with the linear control approach. In Sec. VIII, a new momentum dumping method that does not use torque-generating thrusters is presented.

\section{Fundamentals of Underactuated Systems}

We have proven in [19] that a fully decentralized control law designed from a single-tethered spacecraft can also stabilize arbitrarily large circular arrays of tethered spacecraft, including a two-spacecraft configuration. Furthermore, due to the hierarchical combination, the dynamics of a three-inline configuration reduce to those of the single-tethered systems if the center spacecraft becomes exponentially stabilized by a simple independent control law (see Fig. 1). Consequently, we first focus on control of an underactuated single-tethered system (see Fig. 2c), and then discuss decentralization and decoupling in Sec. $\overline{\mathrm{VI}}$. To that end, we proceed to illustrate the dynamics and challenges of the underactuated singletethered system.

\section{A. Underactuated Single-Tethered Systems}

Underactuated mechanical systems are characterized by fewer actuators than degrees of freedom (DOF) or configuration variables and are encountered in a wide range of applications, such as walking robots, aerospace vehicles [4], and nonholonomic systems [2]. Popular 2-DOF examples include the acrobot (Fig. 2a) and the pendubot (Fig. 2b), where the control input is available only to one joint variable. In contrast, the single-tethered system shown in Fig. 2c is underactuated via input coupling. This paper also serves the purpose of proposing the single-tethered system as another underactuated control benchmark problem. We also attempt to make a connection between the single-tethered system, which is a fundamental building block for constructing multispacecraft arrays, and a two-link planar robot, which has been a representative example in nonlinear control theory.

The equations of motion for the single-tethered system under the torque actuator only $(u \neq 0, F=0)$ becomes [13]

$$
\mathbf{M}_{1}(\phi)\left(\begin{array}{c}
\ddot{\theta} \\
\ddot{\phi}
\end{array}\right)+\mathbf{C}_{\mathbf{1}}(\phi, \dot{\theta}, \dot{\phi})\left(\begin{array}{c}
\dot{\theta} \\
\dot{\phi}
\end{array}\right)=\left(\begin{array}{l}
u \\
u
\end{array}\right)
$$

where

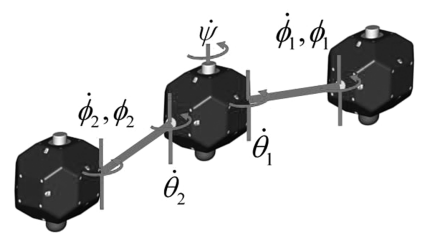

a) Three-inline configuration with a hierarchical combination

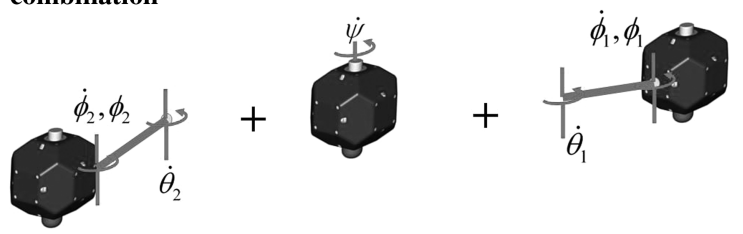

b) Decoupled into two independent single-tethered systems and a center spacecraft

Fig. 1 Three-spacecraft array decoupled into three subsystems (see [19]).

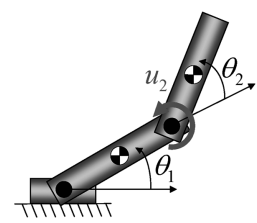

a) Acrobot

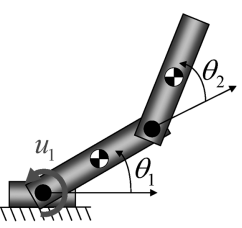

b) Pendubot

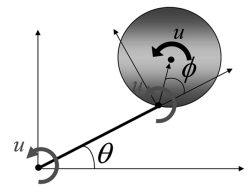

c) Tethered-spacecraft without thrusters
Fig. 2 Three representative cases of underactuated two-link mechanical systems.

$$
\begin{aligned}
& \mathbf{M}_{\mathbf{1}}(\phi)=\left[\begin{array}{cc}
m_{11}(\phi) & m_{12}(\phi) \\
m_{12}(\phi) & m_{22}
\end{array}\right] \\
& =\left[\begin{array}{cc}
I_{r}+m \ell^{2}+2 m r \ell \cos \phi & I_{r}+m r \ell \cos \phi \\
I_{r}+m r \ell \cos \phi & I_{r}
\end{array}\right] \\
& \mathbf{C}_{\mathbf{1}}(\phi, \dot{\theta}, \dot{\phi})=\left[\begin{array}{cc}
c_{11}(\phi, \dot{\phi}) & c_{12}(\phi, \dot{\theta}, \dot{\phi}) \\
c_{21}(\phi, \dot{\theta}) & c_{22}
\end{array}\right] \\
& =\left[\begin{array}{cc}
-m r \ell \sin \phi \dot{\phi} & -m r \ell \sin \phi(\dot{\theta}+\dot{\phi}) \\
+m r \ell \sin \phi \dot{\theta} & 0
\end{array}\right]
\end{aligned}
$$

In the preceding equations, $r, \ell$ denote the satellite's radius and tether length. Also, $I_{r}$ is the moment of inertia about the tether attachment point such that $I_{r}=I_{G}+m r^{2}$, where $I_{G}$ is the moment of inertia about the center of mass (c.m.). In addition, $u$ denotes the torque exerted on the c.m. of the satellite, for example, torque by a reaction wheel assembly (RWA) or diagonal thruster firings. Note that we can derive the preceding equation from the two-link robot manipulator dynamics, by assuming that the mass and moment of inertia of the first link are zero and gravity is absent.

Equation (1) clearly shows that the single input $u$ enters both the configuration variables $\theta$ and $\phi$, as opposed to the acrobot $\tau=$ $\left(\begin{array}{ll}0 & u\end{array}\right)^{T}$ and the pendubot $\tau=\left(\begin{array}{ll}u & 0\end{array}\right)^{T}$. Even though all three cases in Fig. 2 are derived from the two-link manipulator robot, there exists another fundamental difference: the effect of gravity is ignored in the tethered system (see the modeling assumptions in [1]). In particular, underactuated mechanical systems such as the acrobot are, in general, not controllable in the absence of gravity. However, the artificial gravity, induced by the centrifugal force associated with array rotation, plays a crucial role in making the tethered system controllable and stable (see the discussion in [1] ).

\section{B. Challenges of Nonlinear Underactuated Systems}

As mentioned earlier, an underactuated mechanical system is not, in general, exactly input-state feedback linearizable. Its lack of feedback linearizability, along with its complex nonholonomic behavior, characterizes the difficult nonlinear control problem. It has been shown in [20] that the acrobot is not feedback linearizable with static state feedback and nonlinear coordinate transformation. In this section, we derive a similar result for the single-tethered system given in Eq. (1) and Fig. 2c.

Consider a nonlinear system, affine in the control input $u$, with $\mathbf{f}(\mathbf{x})$ and $\mathbf{g}(\mathbf{x})$ being smooth vector fields:

$$
\dot{\mathbf{x}}=\mathbf{f}(\mathbf{x})+\mathbf{g}(\mathbf{x}) u
$$

The system is input-state linearizable [21] in an open set $U$, such that a nonlinear feedback control law $u=\alpha(\mathbf{x}) v+\beta(\mathbf{x})$ and a diffeomorphism $\mathbf{z}=\phi(\mathbf{x})$ transform Eq. (2) to the resultant linear dynamics

$$
\dot{\mathbf{z}}=\mathbf{A z}+\mathbf{b} v
$$

if and only if Eq. (1) dim $\operatorname{span}\left\{g, a d_{f} g, \ldots, a d_{f}^{n-1} g\right\}(\mathbf{x})=n, \forall \mathbf{x} \in$ $U$ in $\mathbb{R}^{n}$, that is, the vector fields are linearly independent and Eq. (2) $\operatorname{span}\left\{g, a d_{f} g, \ldots, a d_{f}^{n-2} g\right\}$ is an involutive distribution on $U$. Note that $a d_{f}^{i} g$ is the iterated Lie bracket [21].

We can easily write the dynamics of the single-tethered system in the first-order form, shown in Eq. (2), by multiplying Eq. (1) with the 
inverse of the inertia matrix $\mathbf{M}_{\mathbf{1}}(\phi)$. The underactuated tethered system in Eq. (1) satisfies the first condition, which corresponds to a controllability test. This result agrees with the linear controllability analysis about the relative equilibria, as discussed in the first paper of this series [1]. The more subtle second condition, derived by Frobenius's theorem, warrants further discussion [22]. To meet the involutivity condition, the following vector fields

$$
\left[g, a d_{f} g\right]\left[g, a d_{f}^{2} g\right]\left[a d_{f} g, a d_{f}^{2} g\right]
$$

must lie in the distribution $\Delta=\operatorname{span}\left\{g, a d_{f} g, a d_{f}^{2} g\right\}$. It is verified in [13] via Mathematica that the matrix constructed by one of the preceding vector fields and $\Delta$ have full rank of four. This, in turn, implies that they do not lie in the distribution $\Delta$ (all vectors are independent). As a result, similar to the acrobot, the underactuated single-tethered system fails the involutivity test, and hence is not input-state feedback linearizable.

Nevertheless, there might exist an output function to render inputoutput feedback linearizability. Finding such an output function is not trivial, and additional work is required to ensure that the associated zero dynamics are stable. This is one of the reasons that designing an efficient control law of a large class of underactuated systems is generally an open problem. In Sec. IV, we introduce a nonlinear diffeomorphism that permits model reduction and simple feedback linearization about the transformed state vector, inspired by the following normal forms $[\underline{4}, \underline{8}]$

\section{Normal Forms for Underactuated Systems}

Olfati-Saber [4,8] developed cascade normal forms for underactuated mechanical systems, based upon the mechanical symmetry. Normal forms can be further classified into triangular normal forms and nontriangular forms. Both strict-feedback and strict-feedforward systems are called "triangular" by analogy with linear systems. In particular, a strict-feedback system permits a systematic nonlinear control design called backstepping.

Let us consider the dynamics similar to the acrobot such that the input is applied only to the shape variable $q_{2}$ :

$$
\begin{aligned}
& m_{11}\left(q_{2}\right) \ddot{q}_{1}+m_{12}\left(q_{2}\right) \ddot{q}_{2}+h_{1}\left(q_{1}, q_{2}, \dot{q}_{1}, \dot{q}_{2}\right)=0 \\
& m_{21}\left(q_{2}\right) \ddot{q}_{1}+m_{22}\left(q_{2}\right) \ddot{q}_{2}+h_{2}\left(q_{1}, q_{2}, \dot{q}_{1}, \dot{q}_{2}\right)=\tau
\end{aligned}
$$

where the dynamics are kinetically symmetric with respect to $q_{2}$, such that $m_{i j}(\mathbf{q})=m_{i j}\left(q_{2}\right)$. Similar to the partial linearization, there exists an invertible change of control input $\tau=\alpha(\mathbf{q}) u+\beta(\mathbf{q}, \dot{\mathbf{q}})$, which transforms the dynamics into

$$
\begin{gathered}
\dot{q}_{1}=p_{1} \\
\dot{p}_{1}=-m_{11}^{-1}\left(q_{2}\right) h_{1}\left(q_{1}, q_{2}, p_{1}, p_{2}\right)-m_{11}^{-1}\left(q_{2}\right) m_{12}\left(q_{2}\right) u \\
\dot{q}_{2}=p_{2} \quad \dot{p}_{2}=u
\end{gathered}
$$

Because the linearization was performed on the actuated variable $q_{2}$, such a change of control is called collocated partial feedback linearization. Olfati-Saber $[\underline{4}, \underline{8}]$ introduces a diffeomorphism, transforming the preceding equation into a strict-feedback form:

$$
\dot{z}_{1}=m_{11}^{-1}\left(\xi_{1}\right) z_{2} \quad \dot{z}_{2}=g\left(z_{1}, \xi_{1}\right) \quad \dot{\xi}_{1}=\xi_{2} \quad \dot{\xi}_{2}=u
$$

where $g(\cdot, \cdot)$ is the gravity term. Unfortunately, the single-tethered system shown in Fig. 2c does not permit the same strict-feedback form due to its input coupling and the lack of such a gravity function. Nevertheless, in Sec. IV, we show that the same transformation yields a useful coordinate transformation permitting feedback linearization and backstepping control design for the reduced variables $z_{1}$ and $z_{2}$.

We can also show that the pendubot in Fig. $2 \mathrm{~b}$ can be transformed into a cascade nonlinear system in nontriangular quadratic normal form by a similar transformation:

$$
\begin{gathered}
\dot{z}_{1}=m_{21}^{-1}\left(\xi_{1}\right) z_{2} \\
\dot{z}_{2}=g\left(z_{1}, \xi_{1}\right)+\left(z_{2}, \xi_{2}\right) \pi\left(\xi_{1}\right)\left(z_{2}, \xi_{2}\right)^{T} \\
\dot{\xi}_{1}=\xi_{2} \quad \dot{\xi}_{2}=u
\end{gathered}
$$

Stabilization of a nontriangular form, addressed in $[\underline{4}, \underline{8}]$, is in general much more difficult than that of a triangular form. For example, backstepping or forwarding [4] is not applicable. Even though the single-tethered dynamics in Fig. 2 can be transformed into a nontriangular form, such a method is not pursued in this paper due to the challenge associated with a nontriangular form. Instead, we apply feedback linearization and backstepping to the reduced system by using a transformation similar to Eq. (7), in addition to partial feedback linearization.

\section{Partial Feedback Linearization}

The present section describes a nonlinear control law obtained by applying partial feedback linearization. The stability of the zero dynamics is also treated using a new nonlinear stability tool called contraction analysis [23], which has been applied to tethered systems in [19].

\section{A. Collocated Linearization}

The partial feedback linearization technique $[4,6]$ is applied to the following equation, which can be obtained by canceling the input coupling of Eq. (1):

$$
\mathbf{M}_{c}(\phi)\left(\begin{array}{c}
\ddot{\theta} \\
\ddot{\phi}
\end{array}\right)+\left(\begin{array}{c}
h_{1}(\phi, \dot{\theta}, \dot{\phi}) \\
h_{2}(\phi, \dot{\theta})
\end{array}\right)=\left(\begin{array}{l}
0 \\
u
\end{array}\right)
$$

where

$$
\begin{aligned}
& \mathbf{M}_{c}(\phi)=\left[\begin{array}{cc}
m_{c 11}(\phi) & m_{c 12}(\phi) \\
m_{c 21}(\phi) & m_{c 22}
\end{array}\right] \\
& =\left[\begin{array}{cc}
m \ell+m r \cos \phi & m r \cos \phi \\
I_{G}+m r^{2}+m r \ell \cos \phi & I_{G}+m r^{2}
\end{array}\right] \text { and } \\
& \left(\begin{array}{c}
h_{1}(\phi, \dot{\theta}, \dot{\phi}) \\
h_{2}(\phi, \dot{\theta})
\end{array}\right)=\left(\begin{array}{c}
-m r \sin \phi(\dot{\theta}+\dot{\phi})^{2} \\
m r \ell \sin \phi \dot{\theta}^{2}
\end{array}\right)
\end{aligned}
$$

Even though the inertia matrix $\mathbf{M}_{c}(\phi)$ is no longer symmetric, Eq. (9) has eliminated the input coupling, thereby facilitating collocated or noncollocated partial feedback linearization. Equation (9) is physically meaningful, because it can be directly derived by the Newton-Euler formulation, as seen in [13]. Note that the first equation (unactuated part) of Eq. (9) corresponds to the second-order nonholonomic constraint. This system can be partially feedback linearized for $\phi$.

Multiplying the first equation by $m_{c 11}^{-1}(\phi)$, and then inserting the resulting equation for $\ddot{\theta}$ into the second equation yields

$$
\begin{gathered}
\ddot{\theta}=-m_{c 11}^{-1}(\phi) m_{c 12}(\phi) \ddot{\phi}-m_{c 11}^{-1}(\phi) h_{1} \quad \ddot{\phi}=v \\
u=\alpha(\phi) v+\beta(\phi, \dot{\theta}, \dot{\phi}) \\
\alpha(\phi)=m_{c 22}-m_{c 21}(\phi) m_{c 11}^{-1}(\phi) m_{c 12}(\phi) \\
\beta(\phi, \dot{\theta}, \dot{\phi})=h_{2}-m_{c 21}(\phi) m_{c 11}^{-1}(\phi) h_{1}
\end{gathered}
$$

where $v$ is now a new control input to the linearized $\phi$ dynamics. In addition, $\alpha(\phi)$ and $\beta(\phi, \dot{\theta}, \dot{\phi})$ define an invertible change of control between $u$ and $v$.

Because $\phi$ is the actuated variable, it is called collocated partial feedback linearization [6]. Then, we can design the following controller $v$ to asymptotically stabilize $\phi$ dynamics:

$$
v=-D \dot{\phi}-K(\phi-e) \quad e=\tan ^{-1}\left[A\left(\dot{\theta}_{d}-\dot{\theta}\right)\right]
$$

where $D, K$, and $A$ are all positive constants and $\dot{\theta}_{d}$ denotes the desired angular rate of the tethered array. Also note that we chose 
such a definition of $e$, instead of $e=A\left(\dot{\theta}_{d}-\dot{\theta}\right)$, to avoid saturation by accommodating a large value of $\left(\dot{\theta}_{d}-\dot{\theta}\right)$.

Assuming $\dot{e}$ and $\ddot{e}$ are sufficiently close to zero, Eq. (11) makes $\phi$ tend to $e$ asymptotically $(\phi \rightarrow e)$ :

$$
\ddot{\phi}+D \dot{\phi}+K(\phi-e)=0
$$

The rationale behind this choice of $v$ is to balance between the tracking error $\dot{\theta}_{d}-\dot{\theta}$ and the compound pendulum mode $\phi$ by transferring energy between them, similar to []].

\section{B. Analysis of Zero Dynamics}

The zero dynamics are defined to be the internal dynamics of the system when the system output is kept at zero by the input [21]. By analogy with linear systems, a nonlinear system with stable zero dynamics corresponds to a minimum phase system. To investigate the zero dynamics of $\theta$ under this control input $v$ in Eq. (11), the $\theta$ dynamics in Eq. (10) are expanded as

$$
\begin{aligned}
\ddot{\theta} & =-m_{c 11}^{-1}(\phi) m_{c 12}(\phi) \ddot{\phi}-m_{c 11}^{-1}(\phi) h_{1} \\
& =-m_{c 11}^{-1}(\phi)\left[m_{c 12}(\phi) v+h_{1}\right] \\
& =\frac{m r \sin \phi(\dot{\theta}+\dot{\phi})^{2}+m r \cos \phi[D \dot{\phi}+K(\phi-e)]}{m(\ell+r \cos \phi)}
\end{aligned}
$$

If $\phi \rightarrow e$ and $\dot{\phi} \rightarrow 0$, the zero dynamics of $\theta$ become

$$
\ddot{\theta}=-\frac{r \dot{\theta}^{2}}{\ell+r \cos \left\{\tan ^{-1}\left[A\left(\dot{\theta}-\dot{\theta}_{d}\right)\right]\right\}} \sin \left\{\tan ^{-1}\left[A\left(\dot{\theta}-\dot{\theta}_{d}\right)\right]\right\}
$$

If $\ell>r$, which is a reasonable assumption, then $m_{c 11}=m(\ell+r \cos e)>0$. In addition, if a reference array angular rate is a constant step input $\left(\ddot{\theta}_{d}=0, \dot{\theta}_{d} \neq 0\right)$, Eq. (14) reduces to

$$
\frac{\mathrm{d}}{\mathrm{d} t}\left(\dot{\theta}-\dot{\theta}_{d}\right)+L(t) \sin \left\{\tan ^{-1}\left[A\left(\dot{\theta}-\dot{\theta}_{d}\right)\right]\right\}=0
$$

where

$$
L(t)=\frac{r \dot{\theta}^{2}}{\ell+r \cos \left\{\tan ^{-1}\left[A\left(\dot{\theta}-\dot{\theta}_{d}\right)\right]\right\}}>0
$$

for nonzero $\dot{\theta}$.

Let us prove exponential stability of Eq. (15) by applying the partial contraction theory (see the Appendix). The virtual $y$ system

$$
\dot{y}+L(t) \sin \left[\tan ^{-1}(A y)\right]=0
$$

has two particular solutions, namely, $\left(\dot{\theta}-\dot{\theta}_{d}\right)$ and 0 . This $y$ system is contracting (see the Appendix) because its associated Jacobian

$$
-L(t) \cos \left[\tan ^{-1}(A y)\right] \frac{1}{(A y)^{2}+1} A
$$

is negative definite since $L(t)>0, \quad A>0$, and $-(\pi / 2)<\tan ^{-1}(\cdot)<(\pi / 2)$. Hence, all solutions of $y$ tend to each other, which implies $\dot{\theta}$ tends to $\dot{\theta}_{d}$ exponentially.

From Eqs. (11) and (12), the convergence of $e \rightarrow 0$ also implies $\phi \rightarrow 0$, which concludes the stability analysis of the proposed underactuated control law in Eq. (11). Its corresponding $u$ is then defined by the relation between $u$ and $v$ shown in Eq. (10).

\section{Momentum Decoupling and Feedback Linearization of Reduced Models}

Even though exact feedback linearization is not possible for the underactuated tethered system, we show herein that there exists a diffeomorphism such that feedback linearization is made possible with respect to the relative equilibria of a spinning tethered system.

We recall the dynamics of the underactuated single-tethered system with the fixed tether length from Eq. (1):

$$
\begin{aligned}
& \frac{\mathrm{d}}{\mathrm{d} t} \frac{\partial L}{\partial \dot{\theta}}-\frac{\partial L}{\partial \theta}=m_{11} \ddot{\theta}+m_{12} \ddot{\phi}+c_{11} \dot{\theta}+c_{12} \dot{\phi}=u \\
& \frac{\mathrm{d}}{\mathrm{d} t} \frac{\partial L}{\partial \dot{\phi}}-\frac{\partial L}{\partial \phi}=m_{21} \ddot{\theta}+m_{22} \ddot{\phi}+c_{21} \dot{\theta}+c_{22} \dot{\phi}=u
\end{aligned}
$$

where $m_{i j}$ and $c_{i j}$ are defined in Eq. (1).

Following [8] , consider the nonlinear diffeomorphism applying the change of coordinates, such that

$$
z_{1}=\theta+\gamma(\phi) \quad z_{2}=m_{11}(\phi) \dot{\theta}+m_{12}(\phi) \dot{\phi}
$$

where

$$
\gamma=\int_{0}^{\phi} \frac{m_{12}(s)}{m_{11}(s)} \mathrm{d} s=\int_{0}^{\phi} \frac{I_{r}+m r \ell \cos (s)}{I_{r}+m \ell^{2}+2 m r \ell \cos (s)} \mathrm{d} s
$$

As discussed in the first paper of this series [1], the kinetic symmetry with respect to $\theta$ in the absence of a gravitational effect leads to symmetry in mechanics, such that

$$
\frac{\partial K}{\partial \theta}=\frac{\partial L}{\partial \theta}=0
$$

because the corresponding Lagrangian $L$ is independent of $\theta$.

Note that $z_{2}$ is essentially the first generalized angular momentum, such that

$$
z_{2}=\frac{\partial L}{\partial \dot{\theta}}, \quad \dot{z}_{2}=\frac{\mathrm{d}}{\mathrm{d} t} \frac{\partial L}{\partial \dot{\theta}}=\frac{\partial L}{\partial \theta}+u=u
$$

In addition,

$$
\dot{z}_{1}=\dot{\theta}+\frac{m_{12}(\phi)}{m_{11}(\phi)} \dot{\phi}=\frac{m_{11}(\phi) \dot{\theta}+m_{12}(\phi) \dot{\phi}}{m_{11}(\phi)}=\frac{z_{2}}{m_{11}(\phi)}
$$

Incorporating Eqs. (23) and (24), we obtain the following equations of $z_{1}$ and $z_{2}$ :

$$
\dot{z}_{1}=m_{11}^{-1}(\phi) z_{2} \quad \dot{z}_{2}=u
$$

where $m_{11}(\phi)=I_{r}+m \ell^{2}+2 m r \ell \cos \phi$. Note that $m_{11}(\phi)>0, \forall \phi$ since $I_{r}=I_{G}+m r^{2}$.

A closer examination of the definition of $z_{1}$ and $z_{2}$ given in Eq. (20) reveals that $z_{1}$ corresponds to the superposition of two angular variables, $\theta$ and $\phi$, whereas $z_{2}$ is the generalized momentum conjugate to $\theta$.

By differentiating $\dot{z}_{1}$,

$$
\begin{aligned}
\ddot{z}_{1} & =\left[\frac{\partial m_{11}^{-1}(\phi)}{\partial \phi} \dot{\phi} z_{2}\right]+m_{11}^{-1}(\phi) \dot{z}_{2} \\
& =\frac{2 m r \ell \sin \phi}{\left(I_{r}+m \ell^{2}+2 m r \ell \cos \phi\right)^{2}} \dot{\phi} z_{2}+m_{11}^{-1}(\phi) u=v
\end{aligned}
$$

The following definition of the new control input $v$ guarantees exponential convergence of $z_{1}$ to $z_{1 d}$ :

$$
v=\ddot{z}_{1 d}-D\left(\dot{z}_{1}-\dot{z}_{1 d}\right)-K\left(z_{1}-z_{1 d}\right)
$$

where the control gains $K$ and $D$ are positive constants.

For $\phi_{d}=0$ and $\dot{\phi}_{d}=0$, the reference $\dot{z}_{1 d}$ and $\ddot{z}_{1 d}$ can be defined as

$$
\begin{gathered}
\dot{z}_{1 d}=m_{11}^{-1}(\phi) z_{2 d}=m_{11}^{-1}(\phi)\left[m_{11}(\phi) \dot{\theta}_{d}+m_{12}(\phi) \dot{\phi}_{d}\right]=\dot{\theta}_{d} \\
\ddot{z}_{1 d}=\ddot{\theta}_{d}
\end{gathered}
$$

For the error $\left(z_{1}-z_{1 d}\right)$, we are mainly concerned with the array angular rate $\dot{\theta}$ of the spinning tethered array rather than the angle $\theta$. So we consider only the $\gamma(\phi)$ term from the definition of $z_{1}$, such that

$$
z_{1}-z_{1 d} \approx \gamma(\phi)-\gamma\left(\phi_{d}\right)=\gamma(\phi)
$$

where $\gamma(\phi)$ is analytically obtained from the integral in Eq. (20) using Mathematica: 


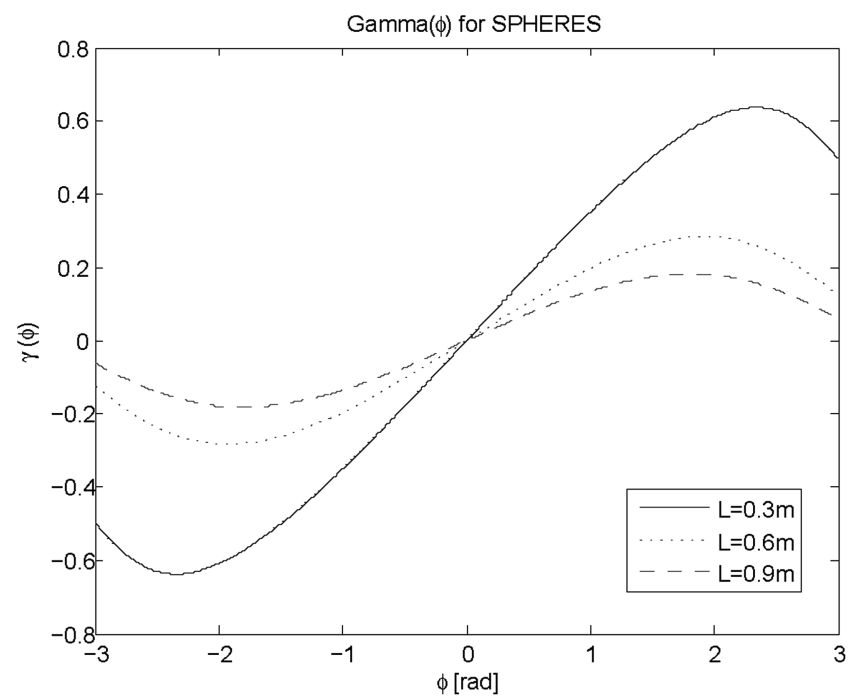

Fig. 3 Plot of $\gamma(\phi)$ using $\ell=(0.3,0.6,0.9 \mathrm{~m})$ and the physical parameters [1] .

$$
\begin{aligned}
\gamma(\phi) & =\int_{0}^{\phi} \frac{m_{12}(s)}{m_{11}(s)} \mathrm{d} s=\int_{0}^{\phi} \frac{I_{r}+m r \ell \cos (s)}{I_{r}+m \ell^{2}+2 m r \ell \cos (s)} \mathrm{d} s=\frac{\phi}{2} \\
& +\frac{m \ell^{2}-I_{r}}{\sqrt{-\left(m \ell^{2}+I_{r}\right)^{2}+4 m^{2} r^{2} \ell^{2}}} \\
& \times \tanh ^{-1}\left(\frac{I_{r}+m \ell(\ell-2 r)}{\sqrt{-\left(m \ell^{2}+I_{r}\right)^{2}+4 m^{2} r^{2} \ell^{2}}} \tan \frac{\phi}{2}\right)
\end{aligned}
$$

Figure 3 plots the function $\gamma(\phi)$ in Eq. (30), which is a monotonic function of $\phi$ within a small range of the compound pendulum mode angle $\phi$. From Eq. (26), the original torque input $u$ can be computed:

$$
u=m_{11}(\phi) v-\frac{2 m r \ell \sin \phi}{m_{11}(\phi)} \dot{\phi} z_{2}
$$

where the new control input $v$ is defined in Eq. (27).

As discussed in Sec. II.B, the original nonlinear system in Eq. (1) is not fully feedback linearizable with respect to its states $\theta, \dot{\theta}, \bar{\phi}, \dot{\phi}$. Nonetheless, the nonlinear control law in Eq. (31), using feedback linearization, is made possible with respect to the reduced variables $z_{1}$ and $z_{2}$. The simulation results in Sec. VII show that the control law in Eq. (31) is particularly efficient for tracking the desired trajectory of $\dot{\theta}_{d}$ while the desired $\phi_{d}$ and $\dot{\phi}_{d}$ are set to zero.

\section{Tracking Control by Backstepping and Contraction Analysis}

Feedback linearization often results in cancellations of useful nonlinearities. To the contrary, backstepping design is more flexible and does not force the designed system to appear linear. We present a backstepping nonlinear control design of the single-tethered system, based upon the strict-feedback cascade normal form introduced in the previous section.

Suppose that the original dynamics in Eq. (2) have a stabilizing control function $u=\alpha(\mathbf{x})$, such that

$$
\frac{\partial V(\mathbf{x})}{\partial \mathbf{x}}[\mathbf{f}(\mathbf{x})+\mathbf{g}(\mathbf{x}) \alpha(\mathbf{x})] \leq-W(\mathbf{x})<0
$$

Since $W(\mathbf{x}): \mathbb{R}^{n} \rightarrow \mathbb{R}$ is negative definite, $\mathbf{x}=\mathbf{0}$ is the global asymptotic equilibrium of the original dynamics in Eq. (2). If $W(\mathbf{x})$ is only positive semidefinite, we can prove the convergence of $W(\mathbf{x})$ to zero via the LaSalle-Yoshizawa theorem [7].

Now, we augment the nonlinear system in Eq. (2) with an integrator

$$
\dot{\mathbf{x}}=\mathbf{f}(\mathbf{x})+\mathbf{g}(\mathbf{x}) \xi \quad \dot{\xi}=u
$$

where $\xi$ is now a virtual control input whose desired value is $\alpha(\mathbf{x})$, which satisfies Eq. (32).

We can design a stabilizing control $\dot{\xi}=u$ for the full system in Eq. (33) via backstepping. Following [7], if $W(\mathbf{x})$ is positive definite, then

$$
V_{a}(\mathbf{x}, \xi)=V(\mathbf{x})+\frac{1}{2}[\xi-\alpha(\mathbf{x})]^{2}
$$

is a control Lyapunov function (CLF) for the full system Eq. (33). In other words, there exists a feedback control $u=\alpha_{a}(\mathbf{x}, \xi)$ that renders $\mathbf{x}=\mathbf{0}, \xi=0$ the globally asymptotically stable equilibrium of Eq. (33) [7]. If $W(\mathbf{x})$ is only positive semidefinite, then we can prove the existence of a feedback control that ensures global boundedness

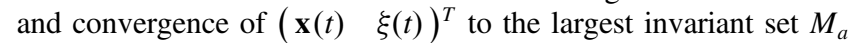
contained in the set $W(\mathbf{x})=0, \xi=\alpha(\mathbf{x})$.

Significant design flexibility is allowed in the backstepping procedure by the choice of the stabilizing function $\alpha(\mathbf{x})$. In other words, a careful choice of $\alpha(\mathbf{x})$ avoids cancellations of useful nonlinearities and allows for additional nonlinear terms to improve transient performance [7].

Let us now turn to the reduced dynamics for the single-tethered system in Eq. (25), where the nonlinear diffeomorphism in Eq. (20) defines $z_{1}$ and $\overline{z_{2}}$. The strict-feedback system, given in Eq. ( $\left.\overline{25}\right)$, regards the variable $\phi$ as an exogenous variable, thereby allowing for backstepping. Let us define the stabilizing function $\alpha\left(z_{1}\right)=-c_{1} z_{1}$, $c_{1}>0$, such that the dynamics

$$
\dot{z}_{1}=m_{11}^{-1}(\phi) \alpha
$$

are asymptotically stable with $V=\frac{1}{2} z_{1}^{2}$. We define the error function $e$, such that

$$
e=z_{2}-\alpha\left(z_{1}\right)=z_{2}+c_{1} z_{1}
$$

and its time derivative is

$$
\dot{e}=\dot{z}_{2}+c_{1} \dot{z}_{1}=u+c_{1} m_{11}^{-1}(\phi)\left(e-c_{1} z_{1}\right)
$$

Suppose that a CLF for $z_{1}$ and $z_{2}$ is $V_{a}=\frac{1}{2} z_{1}^{2}+\frac{1}{2} e^{2}$. Its time derivative should be bounded by the positive definite function $W(\mathbf{x})$ for asymptotic stability:

$$
\begin{aligned}
\dot{V}_{a} & =z_{1} \dot{z}_{1}+e \dot{e}=z_{1} m_{11}^{-1}(\phi)\left(e-c_{1} z_{1}\right) \\
& +e\left[u+c_{1} m_{11}^{-1}(\phi)\left(e-c_{1} z_{1}\right)\right]=-c_{1} m_{11}^{-1}(\phi) z_{1}^{2} \\
& +e\left[u+c_{1} m_{11}^{-1}(\phi) e+\left(1-c_{1}^{2}\right) m_{11}^{-1}(\phi) z_{1}\right]
\end{aligned}
$$

The following $u$ renders $\dot{V}_{a}=-c_{1} m_{11}^{-1}(\phi) z_{1}^{2}-c_{2} e^{2}<0$ with $c_{2}>0$ :

$$
\begin{aligned}
u= & -c_{2} e-c_{1} m_{11}^{-1}(\phi) e+\left(c_{1}^{2}-1\right) m_{11}^{-1}(\phi) z_{1} \\
& =-\left[c_{2} c_{1}+m_{11}^{-1}(\phi)\right] z_{1}-\left[c_{2}+m_{11}^{-1}(\phi) c_{1}\right] z_{2}
\end{aligned}
$$

The closed-loop system in the $\left(z_{1}, e\right)$ coordinates results in

$$
\left(\begin{array}{c}
\dot{z}_{1} \\
\dot{e}
\end{array}\right)=\left[\begin{array}{cc}
-c_{1} m_{11}^{-1}(\phi) & m_{11}^{-1}(\phi) \\
-m_{11}^{-1}(\phi) & -c_{2}
\end{array}\right]\left(\begin{array}{c}
z_{1} \\
e
\end{array}\right)
$$

where $c_{1}$ and $c_{2}$ are positive constants.

The preceding equation shows an interesting property. The system matrix is uniformly (independent of time) negative definite due to the skew-symmetric off-diagonal terms and the positive $m_{11}(\phi)$ term. Possessing such a negative-definite system matrix is an important characteristic of backstepping design [7]. It is emphasized that a similar discussion automatically leads to contraction analysis (see the Appendix). The resulting equation for $\left(z_{1}, e\right)$ in Eq. (40) is contracting due to its uniformly negative-definite Jacobian, hence $(0$, $0)$ is an exponentially stable equilibrium of $\left(z_{1}, e\right)$.

Because we are more interested in tracking control of the underactuated system, the following tracking control law is 
suggested based upon Eqs. (39) and (40):

$$
u=-\left[c_{2} c_{1}+m_{11}^{-1}(\phi)\right] z_{1}-\left[c_{2}+m_{11}^{-1}(\phi) c_{1}\right]\left(z_{2}-z_{2 d}\right)+\dot{z}_{2 d}
$$

where $z_{2 d}=m_{11}(\phi) \dot{\theta}_{d}$ and $\dot{z}_{2 d}=m_{11}(\phi) \ddot{\theta}_{d}$ due to $\phi_{d}=0, \dot{\phi}_{d}=0$. Because we focus on the angular rate $\dot{\theta}, z_{1 d}$ is defined such that $z_{1}-z_{1 d}=z_{1}$ and $\dot{z}_{1 d}=m_{11}^{-1}(\phi) z_{2 d}$. Additionally, we set $e_{d}=z_{2 d}+$ $c_{1} z_{1 d}$ and $\dot{e}_{d}=\dot{z}_{2 d}+c_{1} \dot{z}_{1 d}$. Then, the control law in Eq. (41) leads to the closed-loop system of the virtual variables $y_{1}$ and $y_{2}$, which has

$$
\left(\begin{array}{l}
y_{1} \\
y_{2}
\end{array}\right)=\left(\begin{array}{c}
z_{1}-z_{1 d} \\
e-e_{d}
\end{array}\right) \text { and }\left(\begin{array}{l}
y_{1} \\
y_{2}
\end{array}\right)=\left(\begin{array}{l}
0 \\
0
\end{array}\right)
$$

as particular solutions. Its virtual displacement equation results in

$$
\left(\begin{array}{l}
\delta \dot{y}_{1} \\
\delta \dot{y}_{2}
\end{array}\right)=\left[\begin{array}{cc}
-c_{1} m_{11}^{-1}(\phi) & m_{11}^{-1}(\phi) \\
-m_{11}^{-1}(\phi) & -c_{2}
\end{array}\right]\left(\begin{array}{l}
\delta y_{1} \\
\delta y_{2}
\end{array}\right)
$$

This is contracting because the symmetric part of its Jacobian matrix

$$
\left[\begin{array}{cc}
-c_{1} m_{11}^{-1}(\phi) & 0 \\
0 & -c_{2}
\end{array}\right]
$$

is uniformly negative definite (see the Appendix for details of contraction theory). Hence, all solutions of $y_{1}$ and $y_{2}$ tend to each other, resulting in $\dot{\theta} \rightarrow \dot{\theta}_{d}$ and $\phi, \dot{\phi} \rightarrow 0$ from the definition of $z_{1}, z_{2}$, and $e$. Furthermore, the contraction rate of $z_{1}$ is proportional to $c_{1}$, whereas $c_{2}$ independently determines the contraction rate of $e$. This indicates that we can properly tune the gains $c_{1}$ and $c_{2}$ for desired tracking performance of $z_{1}$ and $z_{2}$, respectively. To maintain the same convergence rate for $z_{2}$ over various tether lengths, we can set $c_{2} \rightarrow c_{2} m_{11}^{-1}(\phi)$.

\section{Decentralized Control For Multivehicle Systems}

Following the model reduction technique introduced in [19], we show herein that a fully decentralized control law designed from the underactuated single-tethered system can stabilize a multivehicle tethered array. The decentralized controller will enable simple independent control of each satellite by eliminating the need for exchanging individual state information. This will significantly simplify both the control algorithm and hardware implementation, as well as eliminate any possibility of performance degradation due to noisy and delayed communications.

Consider a two-spacecraft array with only torque input $\left(u_{1}, u_{2}\right)$, as illustrated in Fig. 4:

$$
\mathbf{M}_{\mathbf{2}}\left(\phi_{1}, \phi_{2}\right)\left(\begin{array}{c}
\ddot{\theta} \\
\ddot{\phi_{1}} \\
\ddot{\phi_{2}}
\end{array}\right)+\mathbf{C}_{\mathbf{2}}\left(\phi_{1}, \phi_{2}, \dot{\theta}, \dot{\phi}_{1}, \dot{\phi}_{2}\right)\left(\begin{array}{c}
\dot{\theta} \\
\dot{\phi}_{1} \\
\dot{\phi}_{2}
\end{array}\right)=\left(\begin{array}{c}
u_{1}+u_{2} \\
u_{1} \\
u_{2}
\end{array}\right)
$$

where

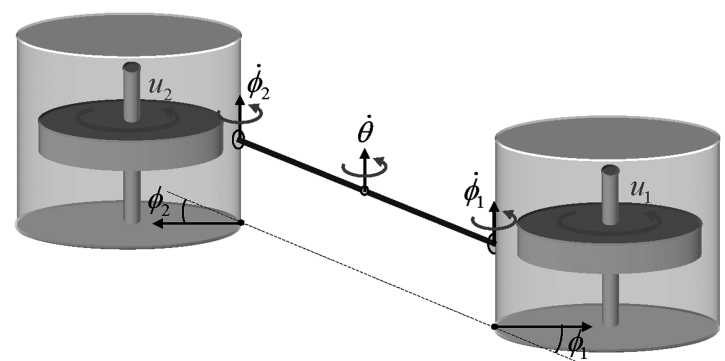

Fig. 4 Two-spacecraft tethered system with a reaction wheel, depicted on the rotation plane. The $\phi_{1}$ and $\phi_{2}$ angles indicate the compound pendulum modes.

$$
\begin{gathered}
\mathbf{M}_{2}\left(\phi_{1}, \phi_{2}\right)=\left[\begin{array}{ccc}
m_{11}\left(\phi_{1}\right)+m_{11}\left(\phi_{2}\right) & m_{12}\left(\phi_{1}\right) & m_{12}\left(\phi_{2}\right) \\
m_{12}\left(\phi_{1}\right) & m_{22} & 0 \\
m_{12}\left(\phi_{2}\right) & 0 & m_{22}
\end{array}\right] \\
\mathbf{C}_{\mathbf{2}}\left(\phi_{1}, \phi_{2}, \dot{\theta}, \dot{\phi}_{1}, \dot{\phi}_{2}\right) \\
=\left[\begin{array}{ccc}
c_{11}\left(\phi_{1}, \dot{\phi}_{1}\right)+c_{11}\left(\phi_{2}, \dot{\phi}_{2}\right) & c_{12}\left(\phi_{1}, \dot{\theta}, \dot{\phi}_{1}\right) & c_{12}\left(\phi_{2}, \dot{\theta}, \dot{\phi}_{2}\right) \\
c_{21}\left(\phi_{1}, \dot{\theta}\right) & c_{22} & 0 \\
c_{21}\left(\phi_{2}, \dot{\theta}\right) & 0 & c_{22}
\end{array}\right]
\end{gathered}
$$

and $m_{i j}$ and $c_{i j}$ are defined in the single-tethered dynamics in Eq. (1).

We can proceed to prove the stability of the nonlinear decentralized control law introduced in Sec. IV. The proof entails showing that such a decentralized control law can de facto synchronize the two compound pendulum mode angles: $\phi_{1}$ and $\phi_{2}$ for the two-spacecraft system. Recall that the second and third rows of Eq. (45) are the independent dynamics for $\phi_{1}$ and $\phi_{2}$, respectively:

$$
\begin{aligned}
& \left(I_{r}+m r \ell \cos \phi_{1}\right) \ddot{\theta}+I_{r} \ddot{\phi}_{1}+m r \ell \dot{\theta}^{2} \sin \phi_{1}=u_{1} \\
& \left(I_{r}+m r \ell \cos \phi_{2}\right) \ddot{\theta}+I_{r} \ddot{\phi}_{2}+m r \ell \dot{\theta}^{2} \sin \phi_{2}=u_{2}
\end{aligned}
$$

where the decentralized control law $u_{i}, i=1,2$ from Eq. (31) can be written as

$$
\begin{aligned}
u_{i} & =m_{11}\left(\phi_{i}\right)\left[\ddot{\theta}_{d}-D\left(\dot{\theta}-\dot{\theta}_{d}\right)\right]-D m_{12}\left(\phi_{i}\right) \dot{\phi}_{i} \\
& -K m_{11}\left(\phi_{i}\right) \gamma\left(\phi_{i}\right)-\frac{2 m r \ell \sin \phi_{i}}{m_{11}\left(\phi_{i}\right)} \dot{\phi}_{i} z_{2}\left(\phi_{i}\right)
\end{aligned}
$$

Because the $\phi$ angle is stabilized ( $\phi \rightarrow 0$ ), assume that $\phi$ and $\dot{\phi}$ are sufficiently small such that $m_{11}(\phi) \approx m_{11}(0), \cos \phi \approx 1$, and $\sin \phi \dot{\phi} \approx 0$. Then, the closed-loop dynamics in Eq. (47) can be simplified as

$$
\begin{aligned}
& I_{r} \ddot{\phi}_{1}+D m_{12}\left(\phi_{1}\right) \dot{\phi}_{1}+K m_{11}\left(\phi_{1}\right) \gamma\left(\phi_{1}\right)+m r \ell \dot{\theta}^{2} \sin \phi_{1}=g(t) \\
& I_{r} \ddot{\phi}_{2}+D m_{12}\left(\phi_{2}\right) \dot{\phi}_{2}+K m_{11}\left(\phi_{2}\right) \gamma\left(\phi_{2}\right)+m r \ell \dot{\theta}^{2} \sin \phi_{2}=g(t)
\end{aligned}
$$

where the common excitation input is defined as

$$
g(t)=-\left(I_{r}+m r \ell\right) \ddot{\theta}+m_{11}(0)\left[\ddot{\theta}_{d}-D\left(\dot{\theta}-\dot{\theta}_{d}\right)\right]
$$

Also, note that $m_{11}(\phi)>0 \forall \phi$ and $m_{12}(\phi)>0$ for $|\phi|<\pi / 2$.

Consider the virtual dynamics of $y$ that has $\phi_{1}$ and $\phi_{2}$ as its particular solutions:

$$
I_{r} \ddot{y}+D m_{12}(y) \dot{y}+K m_{11}(y) \gamma(y)+m r \ell \dot{\theta}^{2} \sin y=g(t)
$$

The preceding dynamics are contracting $(\delta y \rightarrow 0)$ with $D>0$ and $K>0$ in the region $|\phi|<\pi / 2$, indicating that any solutions of $y$ converge to each other. This in turn implies that $\phi_{1}$ tends to $\phi_{2}$ exponentially fast. Once $\phi_{1} \rightarrow \phi_{2}$, it is straightforward to show that the equation of motion for two spacecraft in Eq. (45) reduces to the superposition of the reduced variables $z_{1}$ and $z_{2}$ for each spacecraft. As a result, a decentralized control law designed from the singletethered dynamics not only stabilizes the coupled two-spacecraft dynamics, but also synchronizes the compound pendulum mode angles $\phi_{1}$ and $\phi_{2}$. Following the discussion in [19], the preceding result can be extended to a triangular configuration and a three-inline configuration. In particular, due to the hierarchical combination, the dynamics of a three-inline configuration reduce to those of the singletethered systems if the center spacecraft becomes exponentially stabilized by a simple independent control law. In other words, the preceding result shows that implementing an underactuated control law based on the single-tethered dynamics in Fig. 2 ensures the stability of the rotation rate and the relative motions in an inline threespacecraft array (see the discussion in [19]). 


\section{Simulation Results}

We compare the tracking performance of the two nonlinear underactuated control laws, introduced in Secs. IV and $\underline{\mathrm{V}}$, with that of the linear quadratic regulator (LQR) control. In addition, we validate the effectiveness of a decentralized nonlinear underactuated control law for a two-spacecraft configuration, as well as a three-spacecraft inline configuration, thereby further extending the theory in [19] to underactuated tethered systems.

\section{A. Comparison with Linear Quadratic Regulator Control}

We illustrate that the nonlinear control approach is superior to the linear control approach in tracking a time-varying trajectory. For each simulation, the desired angular rate of the array $\dot{\theta}_{d}$ is given as

$$
\begin{gathered}
\dot{\theta}_{d}=0.25+0.02 e^{-\tau t}[1-\cos (2 \pi f t)] \\
\ddot{\theta}_{d}=0.02 e^{-\tau t}\{2 \pi f[\sin (2 \pi f t)]-\tau[1-\cos (2 \pi f t)]\}
\end{gathered}
$$

where $f=0.01, \tau=0.02$. The control law is also required to minimize the compound pendulum modes such that $\phi_{d}, \dot{\phi}_{d}=0$ while trying to follow $\dot{\theta}_{d}$.

The initial conditions are defined as $\dot{\theta}_{0}=0.25 \mathrm{rad} / \mathrm{s}, \phi_{0}=$ $0.1 \mathrm{rad}$, and $\dot{\phi}_{0}=-0.05 \mathrm{rad} / \mathrm{s}$. The physical parameters used in the simulations are selected from the actual values of the SPHERES test bed on the new air-bearing carriage described in the first paper of this series [1]. The radius of SPHERES $r$ is $0.15 \mathrm{~m}$, the mass of SPHERES with the air-bearing carriage $m$ is $20.346 \mathrm{~kg}$, and the moment of inertia $I$ is $0.178 \mathrm{kgm}^{2}$. The tether length $\ell$ is either 0.5 or $1 \mathrm{~m}$.

Figure 5 shows the performance of the nonlinear tracking control using the feedback linearization of the reduced variables in Eq. (31). The gains are defined as $K=1$ and $D=2$. The nonlinear control is denoted by NLFL (nonlinear tracking control using feedback linearization) and compared with the LQR control. For the LQR control, the $Q$ weighting matrix is $\operatorname{diag}\left(\left[\begin{array}{lll}1 & 5 & 1\end{array}\right]\right)$ and the nominal angular rate of $\dot{\theta}=\omega=0.25 \mathrm{rad} / \mathrm{s}$ are used. The simulation clearly indicates that the nonlinear control is superior to the LQR control in terms of tracking error. Both control approaches turn out to be equally efficient in minimizing the compound pendulum mode $(\phi, \dot{\phi})$. As the tether length $\ell$ increases from $0.5 \mathrm{~m}$ (Fig. $5 \mathrm{a}$ ) to $1.0 \mathrm{~m}$ (Fig. 5b), the tracking performance for the LQR control degrades, even though the change in the tether length was taken into account in computing the optimal LQR gains. This degradation in the performance of the LQR control has to do with the fact that the underactuated tethered system becomes less controllable as the tether length increases (see the controllability analysis in [1]). In contrast, the nonlinear control achieves the same level of performance regardless of the tether length variation. In addition, cumbersome gain scheduling is not required for the nonlinear control approach.

Likewise, Fig. 6 represents the performance of the nonlinear tracking control law derived by the backstepping design approach (NLBS) in Eq. (41). The gains used for this simulation are $c_{1}=4$ and $c_{2}=2$. The figures clearly indicate that the nonlinear control approach using backstepping demonstrates more efficient tracking performance than the LQR control, whose performance deteriorates as the tether length increases.

\section{B. Two-Spacecraft System}

We simulate the proposed decentralized underactuated control law in Eqs. (31) and (요) for the two-spacecraft tethered system shown in Fig. 4. The desired trajectory $\dot{\theta}_{d}$ is defined as in the previous section. Because the total tether length in Eq. (45) is $2 \ell, \ell=1 \mathrm{~m}$ is used. All other physical parameters of the SPHERES satellite remain the same as in the previous section, including the control gains $(K=1$ and $D=2)$. The initial conditions are defined as $\dot{\theta}(0)=0.25 \mathrm{rad} / \mathrm{s}$, $\dot{\psi}(0)=0.25 \mathrm{rad} / \mathrm{s}, \quad \phi_{1}(0)=0.1 \mathrm{rad}, \quad \dot{\phi}_{1}(0)=0 \mathrm{rad} / \mathrm{s}, \quad \phi_{2}(0)=$ $-0.1 \mathrm{rad}$, and $\dot{\phi}_{2}(0)=0 \mathrm{rad} / \mathrm{s}$. As illustrated in Fig. 7, the control law works efficiently to follow the trajectory $\dot{\theta}_{d}$ while minimizing the compound pendulum mode $\left(\phi_{1}\right.$ and $\left.\phi_{2}\right)$. It should be stressed that the
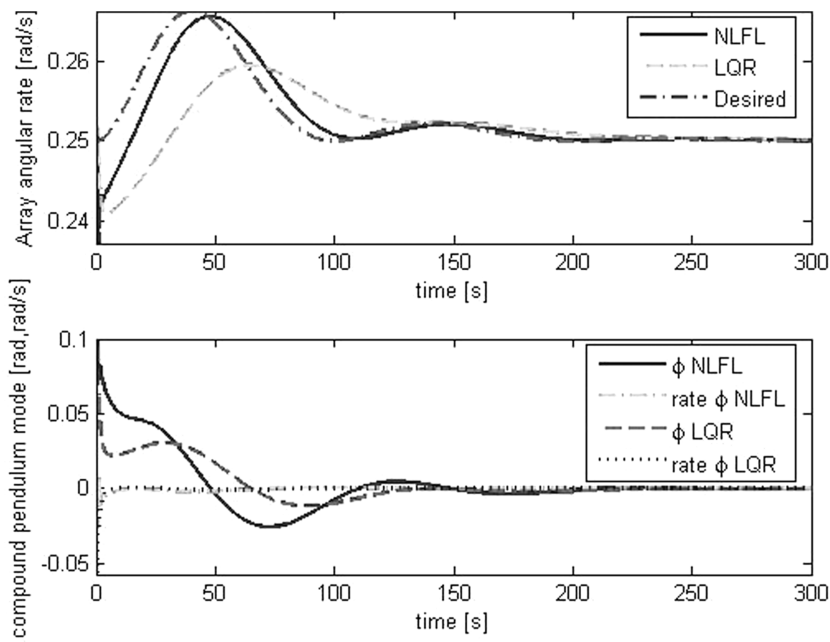

a) Tether length $=0.5 \mathrm{~m}$
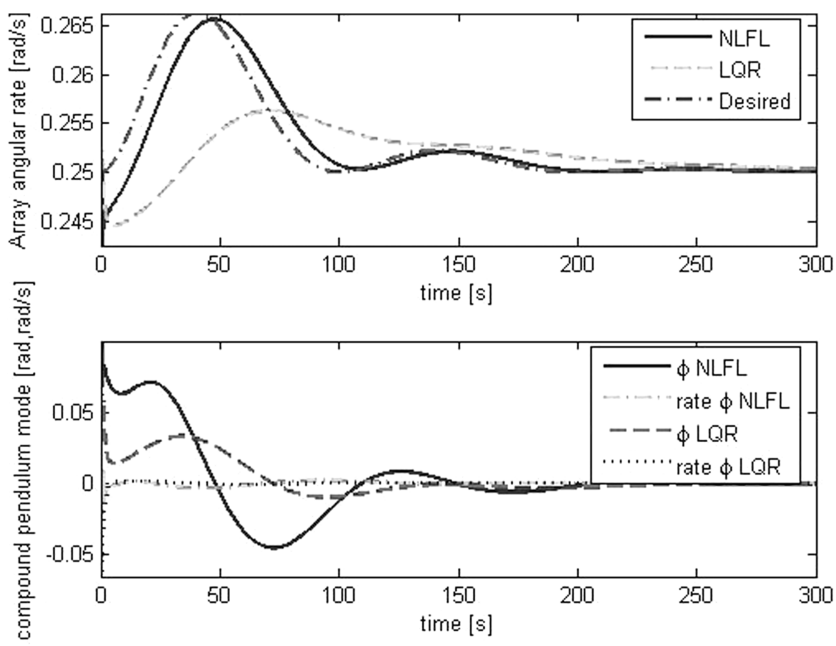

b) Tether length $=1.0 \mathrm{~m}$

Fig. 5 Nonlinear tracking control using the feedback linearization of the reduced variable (NLFL) in Sec. IV and the LQR control.

control law makes both the compound pendulum mode angles synchronize exponentially (i.e., $\phi_{1} \rightarrow \phi_{2}$ ) due to the discussion in Sec. VI. We can easily find why the state responses of the twospacecraft system are similar to those of the single-tethered system in the previous section. Once the two individual spacecraft are synchronized, they behave as one unified closed-loop dynamic of the single-tethered system.

\section{Three-Spacecraft Inline Configuration}

Following the discussion in Sec. VI, we also investigate if the proposed method of designing a nonlinear underactuated control law from the decoupled single-tethered dynamics can be applied to the three-inline configuration shown in Fig. 1. The equations of motion are given in [19]. Figure $\underline{8}$ shows a simulation result obtained by the same underactuated control law in Eq. (48) for the two outlying spacecraft in the linear three-spacecraft tethered array. For the center spacecraft, a simple linear control law, $u_{0}=-0.228\left(\dot{\psi}-\dot{\theta}_{d}\right)$, is used for a spin-up operation. The nonlinear control gains are $K=0.5$ and $D=2$, whereas the only nonzero initial conditions are given as $\dot{\psi}=0$ and $\phi_{1}=0.05 \mathrm{rad}$. During the spin-up maneuver of $\dot{\psi}$ from 0.25 to $0.27 \mathrm{rad} / \mathrm{s}$, the compound pendulum modes $\phi_{1}$ and $\phi_{2}$ get excited due to the coupling motions of the underactuated dynamics. Eventually, the compound pendulum modes $\phi_{1}$ and $\phi_{2}$ oscillate in sync as they tend to zero (see the discussions in Sec. VI and [19]). In conclusion, the proposed underactuated control law, independently implemented in each spacecraft in a decentralized fashion, also ensures the stability of the closed-loop system. 

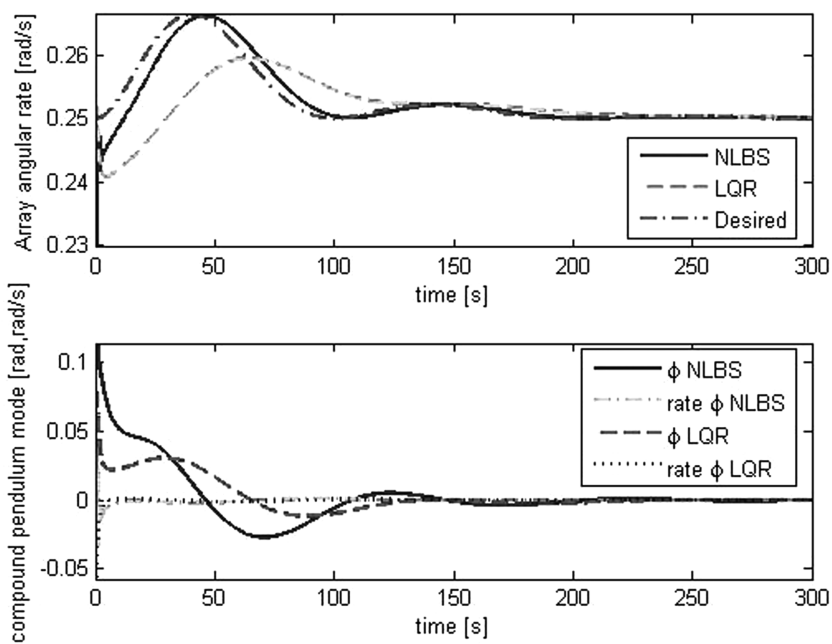

a) Tether length $=0.5 \mathrm{~m}$
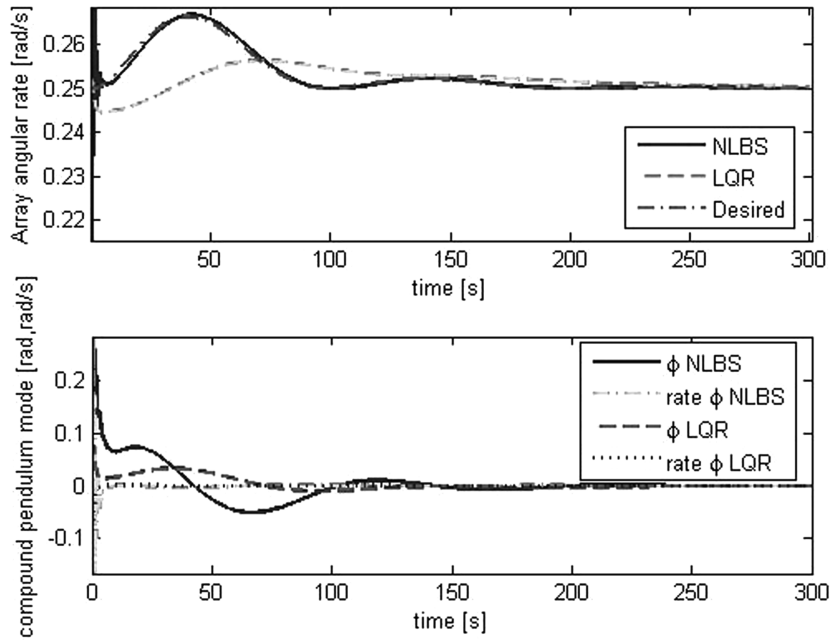

b) Tether length $=1.0 \mathrm{~m}$

Fig. 6 Nonlinear tracking control using backstepping (NLBS) in Sec. $\underline{V}$ and the LQR control.

\section{New Momentum Dumping Method for Saturated Wheels}

If the linear velocity or angular velocity of each spacecraft is held constant, the increase of the tether length and external disturbance torque inevitably lead to the saturation of the wheel speed. For

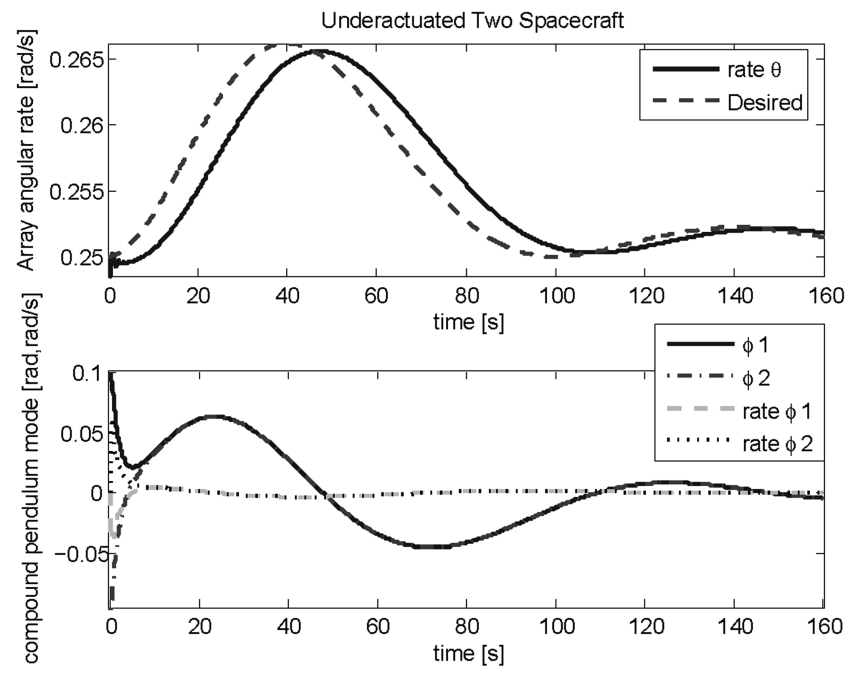

Fig. 7 Simulation result of a decentralized control for Fig. 4 .

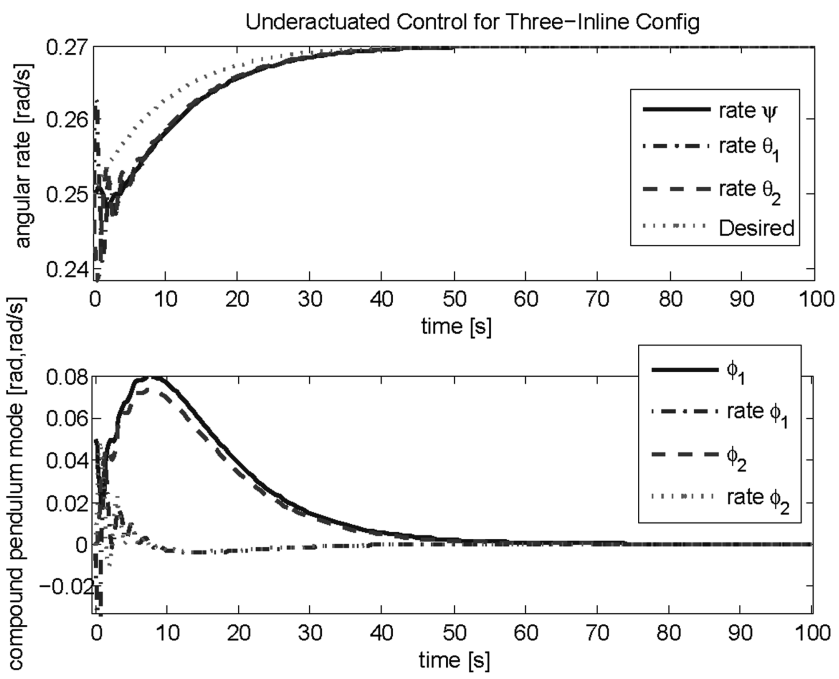

Fig. 8 Simulation result of a decentralized control for a three-inline configuration.

satellites in orbit, a pair of thrusters is conventionally used to dump the angular momentum of the saturated momentum wheel. This section focuses on the issue associated with managing the saturated angular momentum once a tethered array spun by reaction wheels reaches its maximum size. A new technique that can be used to extend the array beyond this size is proposed. The proposed method maintains the desired array spin rate and zero compound pendulum mode during the momentum dumping operation. Maintaining the zero compound pendulum mode without torque-generating thrusters poses a challenge because the reaction wheel, which directly controls the pendulum mode, is decelerated continuously in one direction.

Let us now assume that the tethered formation flight spacecraft shown in Fig. 9 are equipped with only a reaction wheel $u$, a tangential force thruster $F$, and a high-bandwidth translational actuator on the tether $P$ in each spacecraft. The direction of $F$ is perpendicular to the line between the tether attachment point and the c.m. of the spacecraft. It is shown in the first paper [1] that a planar rotating array of tethered spacecraft can control all relevant degrees of freedom using only one reaction wheel $u$ in each spacecraft. Because of the Coriolis force exerted on the spacecraft, a radial motion of the tether can exert torque with respect to the compound pendulum mode $\phi$ in Fig. 9. Oscillatory motions of the tether from the force $P$ can then be used as a means of controlling the pendulum mode. From the linearized dynamics of Eq. (1), the dynamics of $\phi$ is coupled with $\dot{\ell}$ as

$$
\ddot{\phi}+\frac{r \omega^{2}\left[I_{r}+m \ell(2 r+\ell)\right]}{\ell I_{G}} \dot{\phi}=2 \frac{v}{\ell} \dot{\theta}+2 \frac{\omega}{\ell} \dot{\ell}-\frac{1}{m \ell} F+\frac{r+\ell}{I_{G} \ell} u
$$

where $v$ is the nominal tether speed, which is zero here.

Because $\dot{\ell}$ is mainly driven by the force $P$, we can control the compound pendulum mode $\phi$ by exerting the force $P$ on the tether. Such an actuation method can be employed to dump the angular

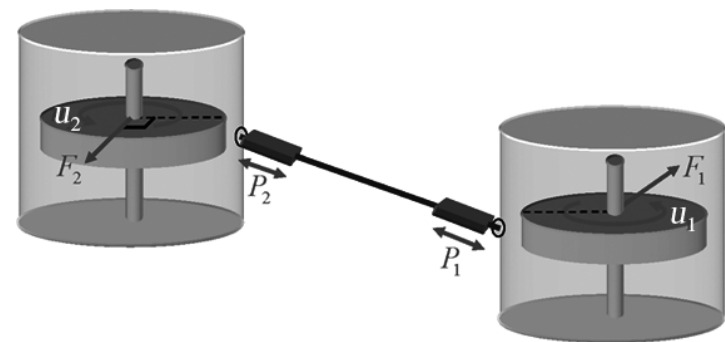

Fig. 9 Two-spacecraft tethered system equipped with a highbandwidth linear actuator on the tether $P$, a reaction wheel $u$, and a tangential thruster $F$ in each spacecraft. 
momentum stored on the reaction wheels. While constantly decelerating the wheel speed, the linear force $P$ on the tether can be exerted in an oscillatory fashion to minimize the associated compound pendulum mode, while the linear thruster $F$ maintains a constant array angular rate. In other words, it is straightforward to show that the system shown in Fig. 9 is fully controllable by $F$ and $P$ when $u$ is not available (see [13]). Hence, the momentum dumping method provides an alternative method for stabilizing the compound pendulum mode during momentum dumping operations.

A simulation of such a momentum dumping operation is presented in Fig. 10. The torque by reaction wheel $u$ is set as $u=-0.01 \mathrm{~N} \cdot \mathrm{m}$ such that the wheel speed can constantly be decelerated to zero. The tangential force thruster $F$ and the translational actuator on the tether $P$ exert the control forces to maintain the same angular rate $\dot{\theta}$ and zero compound pendulum mode $(\phi, \dot{\phi}=0)$ :

$$
\begin{gathered}
F=-10 \phi-10 \dot{\phi}-10\left(\dot{\theta}-\dot{\theta}_{d}\right) \quad u=-0.01 \\
P=-10 \phi-10 \dot{\phi}-10\left(\dot{\theta}-\dot{\theta}_{d}\right)-40\left(\ell-\ell_{d}\right)-40 \dot{\ell}
\end{gathered}
$$

The top plot of Fig. 10a shows the change in the angular momentum of the reaction wheel due to the constant deceleration $u=-0.01 \mathrm{~N} \cdot \mathrm{m}$, while the control forces $F$ and $P$ effectively maintain the control states at the reference points (bottom plot). Figure $10 \mathrm{~b}$ shows that the usage of the linear thruster $F$ to maintain the array angular rate is relatively small. In contrast, large $P$ is required to stabilize the compound pendulum mode in the absence of
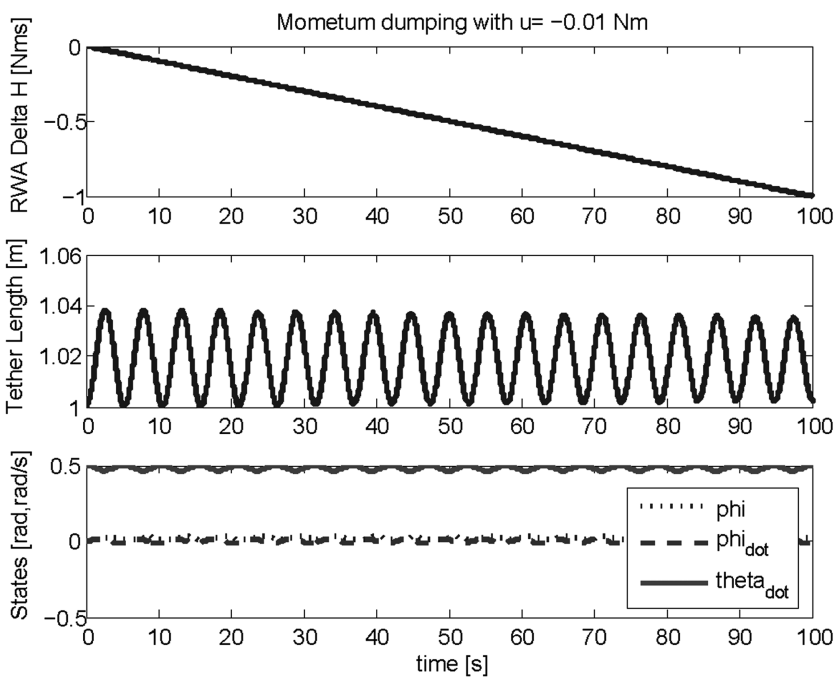

a) Change in the RWA angular momentum and the control states
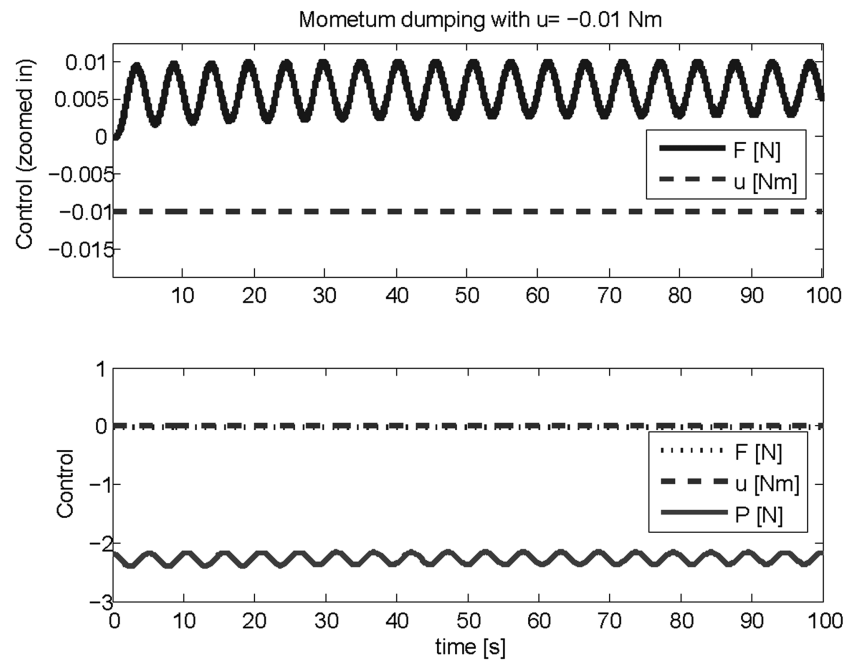

b) Control

Fig. 10 Momentum dumping operation with stabilization. the RWA torque $u$. Small oscillations of both the control states and the tether length are acceptable because no interferometric observation is scheduled during the momentum dumping operation.

\section{Conclusions}

We proposed a new approach for controlling the array spin rate and relative attitude without thrusters by exploiting the coupled dynamics. Such a tethered system without thrusters is underactuated because it has fewer inputs than configuration variables. This work reports the first propellant-free underactuated control results for tethered formation flying spacecraft. Such an underactuated control approach is particularly beneficial to stellar interferometers due to the increased mission life span and reduced optical contamination by exhaust from the thrusters. As discussed in the first paper [1], the effectiveness of the underactuated method decreases as the array size increases. This article also fulfilled the potential of the proposed underactuated strategy by providing a new momentum dumping method that does not use torque-generating thrusters.

In contrast with linear systems, in which an underactuated control law can be synthesized easily, designing a nonlinear controller for nonlinear underactuated systems is a difficult control problem, mainly due to the lack of full state feedback linearizability. In this paper, we derived several nonlinear control laws for spinning tethered systems: partial feedback linearization, feedback linearization via momentum decoupling, and backstepping. Simulation results indicate that the nonlinear control methods are much more efficient in tracking time-varying trajectories than LQR control.

For future work, developing a robust nonlinear underactuated control method that deals with model uncertainties and sensor noise would be an interesting and challenging research topic. Even though the modeling on the two-dimensional rotational plane is justified by the decoupling presented in the first paper of this series [1], it would also be useful to extend such an underactuated control strategy to three-dimensional attitude dynamics. In particular, precessing the array rotation might also be achievable using underactuated tethered systems.

\section{Appendix: Contraction Theory}

We exploit partial contraction theory [23] to prove the stability of coupled nonlinear dynamics. Lyapunov's linearization method indicates that the local stability of the nonlinear system can be analyzed using its differential approximation. What is new in contraction theory is that a differential stability analysis can be made exact, thereby yielding global results on the nonlinear system. A brief review of the results from $[21,23]$ is presented in this section. Readers are referred to these references for detailed descriptions and proofs on the following theorems. Note that contraction theory is a generalization of the classical Krasovskii's theorem [20].

Consider a smooth nonlinear system

$$
\dot{\mathbf{x}}(t)=\mathbf{f}(\mathbf{x}(t), \mathbf{u}(\mathbf{x}, t), t)
$$

where $\mathbf{x}(t) \in \mathbb{R}^{n}$ and $\mathbf{f}: \mathbb{R}^{n} \times \mathbb{R}^{m} \times \mathbb{R}_{+} \rightarrow \mathbb{R}^{n}$. A virtual displacement $\delta \mathbf{x}$ is defined as an infinitesimal displacement at a fixed time: a common supposition in the calculus of variations.

Theorem IX.1: For the system in Eq. (A1), if there exists a uniformly positive definite metric,

$$
\mathbf{M}(\mathbf{x}, t)=\Theta(\mathbf{x}, t)^{T} \Theta(\mathbf{x}, t)
$$

where $\Theta$ is some smooth coordinate transformation of the virtual displacement $\delta \mathbf{z}=\Theta \delta \mathbf{x}$, such that the associated generalized Jacobian $\mathbf{F}$ is uniformly negative definite, that is, $\exists \lambda>0$ such that

$$
\mathbf{F}=\left(\dot{\Theta}(\mathbf{x}, t)+\Theta(\mathbf{x}, t) \frac{\partial \mathbf{f}}{\partial \mathbf{x}}\right) \Theta(\mathbf{x}, t)^{-1} \leq-\lambda \mathbf{I}
$$

then all system trajectories converge globally to a single trajectory exponentially fast regardless of the initial conditions, with a global 
exponential convergence rate of the largest eigenvalues of the symmetric part of $\mathbf{F}$.

Such a system is said to be contracting. The proof is given in [21]. Equivalently, the system is contracting if $\exists \lambda>0$ such that

$$
\dot{\mathbf{M}}+\left(\frac{\partial \mathbf{f}}{\partial \mathbf{x}}\right)^{T} \mathbf{M}+\mathbf{M} \frac{\partial \mathbf{f}}{\partial \mathbf{x}} \leq-2 \lambda \mathbf{M}
$$

It can also be shown that, for a contracting autonomous system of the form $\dot{\mathbf{x}}=\mathbf{f}(\mathbf{x}, \mathbf{u}(\mathbf{x}))$, all trajectories converge to an equilibrium point exponentially fast. In essence, contraction analysis implies that stability of nonlinear systems can be analyzed more simply by checking the negative definiteness of a proper matrix, rather than finding some implicit motion integral as in Lyapunov's theory.

The following theorems are used to derive stability and synchronization of the coupled dynamics systems.

Theorem IX.2, Partial Contraction [23]: Consider a nonlinear system of the form $\dot{\mathbf{x}}=\mathbf{f}(\mathbf{x}, \mathbf{x}, t)$ and assume that the auxiliary system $\dot{\mathbf{y}}=\mathbf{f}(\mathbf{y}, \mathbf{x}, t)$ is contracting with respect to $\mathbf{y}$. If a particular solution of the auxiliary $\mathbf{y}$ system verifies a specific smooth property, then all trajectories of the original $x$ system verify this property exponentially. The original system is said to be partially contracting.

Theorem IX.3, Synchronization [23]: Consider two coupled systems. If the dynamics equations verify

$$
\dot{\mathbf{x}}_{1}-\mathbf{f}\left(\mathbf{x}_{1}, t\right)=\dot{\mathbf{x}}_{2}-\mathbf{f}\left(\mathbf{x}_{2}, t\right)
$$

where the function $\mathbf{f}(\mathbf{x}, t)$ is contracting in an input-independent metric, then $\mathbf{x}_{1}$ and $\mathbf{x}_{2}$ will converge to each other exponentially, regardless of the initial conditions. This proof can be derived by Theorem IX.2.

\section{Acknowledgments}

The authors would like to gratefully acknowledge the NASA Goddard Space Flight Center (Contract Monitor, David Leisawitz) for both financial and technical support for the Massachusetts Institute of Technology-Space Systems Laboratory and Payload Systems SPHERES Tether program. The authors also thank the associate editor (Jesse Leitner) and the anonymous reviewer for their constructive feedback.

\section{References}

[1] Chung, S.-J., Slotine, J.-J. E., and Miller, D. W., "Propellant-Free Control of Tethered Formation Flight, Part 1: Linear Control and Experimentation," Journal of Guidance, Control, and Dynamics, Vol. 31, No. 3, May-June 2008, pp. 571-584. doi: $10.2514 / 1.32188$

[2] Bloch, A. M., Baillieul, J., Crouch, P., and Marsden, J., Nonholonomic Mechanics and Control, Springer-Verlag, New York, 2003.

[3] Bullo, F., and Lewis, A. D., Geometric Control of Mechanical SystemsModeling Analysis, and Design for Simple Mechanical Control Systems, Texts in Applied Mathematics, Springer-Verlag, New York, 2004.

[4] Olfati-Saber, R., "Nonlinear Control of Underactuated Mechanical Systems with Application to Robotics and Aerospace Vehicles," Ph.D. Thesis, Dept. of Electrical Engineering and Computer Science, Massachusetts Inst. of Technology, Cambridge, MA, Feb. 2001, http:// dspace.mit.edu.

[5] Reyhanoglu, M., van der Schaft, A., McClamroch, N. H., and Kolmanovsky, I., "Dynamics and Control of a Class of Underactuated Mechanical Systems," IEEE Transactions on Automatic Control, Vol. 44, No. 9, 1999, pp. 1663-1671. doi: $10.1109 / 9.788533$
[6] Spong, M. W., "Swing Up Control Problem for the Acrobot," IEEE Control Systems Magazine, Vol. 15, No. 1, Feb. 1995, pp. 49-55. doi:10.1109/37.341864

[7] Krstic, K., Kanellakopoulos, I., and Kokotovic, P., Nonlinear and Adaptive Control Design, Wiley, New York, 1995.

[8] Olfati-Saber, R., "Normal Forms for Underactuated Mechanical Systems with Symmetry," IEEE Transactions on Automatic Control, Vol. 47, Feb. 2002, pp. 305-308. doi: $10.1109 / 9.983365$

[9] Wang, W., Yi, J., Zhao, D., and Liu, D., "Design of a Stable SlidingMode Controller for a Class of Second-Order Underactuated Systems," IEEE Proceedings: Control Theory and Applications, Vol. 151, No. 6, Nov. 2004, pp. 683-690. doi:10.1049/ip-cta:20040902

[10] Brown, S. C., and Passino, K. M., "Intelligent Control for an Acrobot," Journal of Intelligent and Robotic Systems: Theory and Applications, Vol. 18, No. 3, March 1997, pp. 209-248. doi:10.1023/A:1007953809856

[11] Zhang, M., and Tarn, T.-J., "Hybrid Switching Control Strategy for Nonlinear and Underactuated Mechanical Systems," IEEE Transactions on Automatic Control, Vol. 48, No. 10, Oct. 2003, pp. 1777 1782.

doi:10.1109/TAC.2003.817911

[12] Woolsey, C., Reddy, C. K., Bloch, A. M., Chang, D. E., Leonard, N. E., and Marsden, J. E., "Controlled Lagrangian Systems with Gyroscopic Forcing and Dissipation," European Journal of Control, Special Issue on Lagrangian and Hamiltonian Methods for Nonlinear Control, Vol. 10, No. 5, Dec. 2004, pp. 478-496. doi:10.3166/ejc. $10.478-496$

[13] Chung, S.-J., "Nonlinear Control and Synchronization of Multiple Lagrangian Systems with Application to Tethered Formation Flight Spacecraft," Sc.D. Thesis, Dept. of Aeronautics and Astronautics, Massachusetts Inst. of Technology, Cambridge, MA, 2007, http:// dspace.mit.edu.

[14] Tsiotras, P., Corless, M., and Longuski, J. M., "Novel Approach to the Attitude Control of Axi-Symmetric Spacecraft," Automatica, Vol. 31, No. 8, 1995, pp. 1099-1112. doi:10.1016/0005-1098(95)00010-T

[15] Tsiotras, P., and Luo, J., "Reduced-Effort Control Laws for Underactuated Rigid Spacecraft," Journal of Guidance, Control, and Dynamics, Vol. 20, No. 6, 1997, pp. 1089-1095.

[16] Tsiotras, P., and Luo, J., "Control of Underactuated Spacecraft with Bounded Inputs," Automatica, Vol. 36, No. 8, 2000, pp. 1153-1169. doi:10.1016/S0005-1098(00)00025-X

[17] Cho, S., and McClamroch, N. H., "Feedback Control of Triaxial Attitude Control Testbed Actuated by Two Proof Mass Devices," Proceedings of 41 st IEEE Conference on Decision and Control, Vol. 1, Inst. of Electrical and Electronics Engineers, New York, 2002, pp. 498 503.

[18] Sanyal, A. K., Shen, J., and McClamroch, N. H., "Control of a Dumbbell Spacecraft using Attitude and Shape Control Inputs Only," Proceedings of American Control Conference, Vol. 2, Inst. of Electrical and Electronics Engineers, New York, 2004, pp. 1014-1018.

[19] Chung, S.-J., Slotine, J.-J. E., and Miller, D. W., "Nonlinear Model Reduction and Decentralized Control of Tethered Formation Flight," Journal of Guidance, Control, and Dynamics, Vol. 30, No. 2, 2007, pp. 390-400. doi:10.2514/1.21492

[20] Slotine, J.-J. E., and Li, W., Applied Nonlinear Control, Prentice-Hall, Upper Saddle River, NJ, 1991.

[21] Lohmiller, W., and Slotine, J. J. E., "On Contraction Analysis for Nonlinear Systems," Automatica, Vol. 34, No. 6, 1998, pp. 683-696. doi:10.1016/S0005-1098(98)00019-3

[22] Murray, R. M., and Hauser, J., "Case Study in Approximate Linearization: The Acrobot Example," Univ. of California, Berkeley Technical Rept. No. UCB/ERL M91/46, 1991.

[23] Wang, W., and Slotine, J. J. E., "On Partial Contraction Analysis for Coupled Nonlinear Oscillators," Biological Cybernetics, Vol. 92, No. 1, 2004, pp. 38-53. doi: $10.1007 / \mathrm{s} 00422-004-0527-\mathrm{x}$ 